\title{
Muscle Contractions Guide Rohon-Beard Peripheral Sensory Axons
}

\author{
Jeremiah D. Paulus, ${ }^{1,2,3}$ Gregory B. Willer, ${ }^{4,5}$ Jason R. Willer, ${ }^{4,5}$ Ronald G. Gregg, ${ }^{4,5}$ and Mary C. Halloran ${ }^{1,2,3}$ \\ Departments of ${ }^{1}$ Zoology and ${ }^{2}$ Anatomy and ${ }^{3}$ Cell and Molecular Biology Training Program, University of Wisconsin, Madison, Wisconsin 53706, and \\ ${ }^{4}$ Department of Biochemistry and Molecular Biology and ${ }^{5}$ Zebrafish Mutant Mapping Facility, University of Louisville, Louisville, Kentucky 40292
}

\begin{abstract}
Multiple molecular cues guide neuronal axons to their targets during development. Previous studies in vitro have shown that mechanical stimulation also can affect axon growth; however, whether mechanical force contributes to axon guidance in vivo is unknown. We investigated the role of muscle contractions in the guidance of zebrafish peripheral Rohon-Beard (RB) sensory axons in vivo. We analyzed several mutants that affect muscle contraction through different molecular pathways, including a new mutant allele of the titin $a$ (pik) gene, mutants that affect the hedgehog signaling pathway, and a nicotinic acetylcholine receptor mutant. We found RB axon defects in these mutants, the severity of which appeared to correlate with the extent of muscle contraction loss. These axons extend between the muscle and skin and normally have ventral trajectories and repel each other on contact. RB peripheral axons in muscle mutants extend longitudinally instead of ventrally, and the axons fail to repel one another on contact. In addition, we showed that limiting muscle movements by embedding embryos in agarose caused similar defects in peripheral RB axon guidance. This work suggests that the mechanical forces generated by muscle contractions are necessary for proper sensory axon pathfinding in vivo.
\end{abstract}

\section{Introduction}

To establish a functioning nervous system, developing axons must interpret a multitude of guidance cues that direct them along specific pathways to their correct targets. Numerous molecular cues have been identified that direct axon outgrowth in vitro and in vivo (Huber et al., 2003; Chilton, 2006; Zou and Lyuksyutova, 2007). Interestingly, even before molecular cues were identified, several in vitro studies demonstrated that mechanical stimulation of neurons also can influence axon initiation, outgrowth, and direction (Bray, 1979, 1984; Heidemann and Buxbaum, 1994; Lamoureux et al., 2002; Anava et al., 2009). Nevertheless, the role of mechanical stimulation in axon guidance in vivo remains unknown.

Several lines of evidence support the idea that mechanical tension may influence axon guidance. In non-neuronal cells, mechanical force induces focal contacts, indicating that tension can modulate cell adhesions and motility (Plopper and Ingber, 1993; Balaban et al., 2001; Riveline et al., 2001; Galbraith et al., 2002). In

Received May 8, 2009; revised Aug. 17, 2009; accepted Sept. 9, 2009.

This work was supported by National Institutes of Health (NIH) Grants R01 NS042228 (M.C.H.) and R01 RR020357 (R.G.G.). The National Science Foundation (NSF) supported acquisition of the Bio-Rad confocal microscope [NSF Grant 9724515 to James Pawley (Department of Zoology, University of Wisconsin, Madison, WI)], and the Olympus confocal was acquired with NIH Shared Instrumentation Grant S10RR023717 [Bill Bement (Department of Zoology, University of Wisconsin)]. The F59 and F310 antibodies developed by Frank E. Stockdale and the $3 \mathrm{~A} 10$ antibody developed by Thomas M. Jessell and Jane Dodd were obtained from the Developmental Studies Hybridoma Bank developed under the auspices of the National Institute of Child Health and Human Development and maintained by Department of Biology of University of lowa (lowa City, IA). We thank Hitoshi Okamoto and Uwe Strähle for DNA constructs, Erik Dent for comments on this manuscript, and numerous other colleagues for in situ probes. We thank Kari Obma and Daniel A. Roberts for technical assistance and Aidan Tesch and Laura Sherrill for fish care.

Correspondence should be addressed to Mary C. Halloran, Department of Zoology, University of Wisconsin, 1117 West Johnson Street, Madison, WI 53706. E-mail: mchalloran@wisc.edu.

DOI:10.1523/JNEUROSCI.2179-09.2009

Copyright $\odot 2009$ Society for Neuroscience $\quad$ 0270-6474/09/2913190-12\$15.00/0 addition, substrate stiffness can affect cell motility and signaling, possibly by increasing tension (Willits and Skornia, 2004; Chan and Odde, 2008; Jiang et al., 2008). Moreover, tension can directly activate mechanosensory ion channels including transient receptor potential (TRP) channels (Nauli et al., 2003; Corey et al., 2004), which recently have been shown to function in axon guidance (Li et al., 1999; Greka et al., 2003; Wang and Poo, 2005). Mechanical stimulation also can affect many of the same intracellular signaling pathways that mediate axon responses to molecular guidance cues. For example, mechanical stimulation can alter levels of cAMP (Chicurel et al., 1998; Meyer et al., 2000; Riveline et al., 2001), which regulate axon responses to guidance molecules (Ming et al., 1997, 1999; Wang and Zheng, 1998; Huber et al., 2003). Similarly, Rho GTPase is an important downstream effector for mechanically induced cell changes (Riveline et al., 2001; Galbraith et al., 2002; Matthews et al., 2006), as well as axon responses to guidance cues (Huber et al., 2003; Gallo and Letourneau, 2004; Kalil and Dent, 2005). These studies raise the intriguing possibility that mechanical tension may cooperate with or influence molecular cues to guide axons in vivo.

Here, we show that muscle contractions guide the pathways and behaviors of overlying sensory axons in vivo. We analyzed several zebrafish mutants that affect muscle contraction via different molecular mechanisms, including a new mutant allele of the titin a (pik) gene, and mutants affecting the hedgehog signaling pathway or acetylcholine receptors. In these mutants, which show either greatly reduced or no muscle contractions, RohonBeard (RB) peripheral axons grew longitudinally instead of ventrally and failed to repel one another on contact. The severity of the axon defects correlated with the extent of muscle contraction loss. Moreover, we show that paralyzing embryos with D-tubocurarine (TC) or limiting muscle movements by embedding embryos 
in agarose cause similar axon guidance defects. Embryos lacking nicotinic acetylcholine receptors showed reduced chondroitin sulfate proteoglycan labeling. Our data suggest that the mechanical forces generated by muscle contraction are critical for proper sensory axon guidance in vivo.

\section{Materials and Methods}

Animals. Zebrafish (Danio rerio) were maintained in a laboratory breeding colony on a 14/10 h light/dark cycle. Embryos were maintained at $28.5^{\circ} \mathrm{C}$ and staged as described previously (Kimmel et al., 1995). Embryo stage was defined as hours postfertilization (hpf) or days postfertilization (dpf). The ttna (pik) $)^{u w 2}$ line was created with ethylnitrosourea as previously described (Haffter et al., 1996). The lama1 (bal) ${ }^{u w 1}$, shha $(s y u)^{t 4}$, gli2a $(y o t)^{t y 17 a}$, smo $(\text { smu })^{b 641}$, and chrnal (nic-1) $)^{b 107}$ fish lines have all been previously described (Westerfield et al., 1990; van Eeden et al., 1996; Schauerte et al., 1998; Barresi et al., 2000; Amsterdam et al., 2004; Paulus and Halloran, 2006). All homozygote mutants were identified by morphology or behavior, where applicable. Controls designated as WT were either wild-type strain $\mathrm{AB}$ or heterozygous mutant siblings, except no heterozygous gli2a $(y o t)^{t y 17 a}$ siblings were used, since this strain has been shown to be partially dominant (Schäfer et al., 2007).

Mapping the J101/ttna mutant locus. The mapping of the J101 mutation was done as previously described (Gregg et al., 2003; Willer et al., 2005). The J101 mutation was generated in wild-type $A B$ fish and propagated by repeated $\mathrm{AB}$ outcrossings. To begin mapping experiments, we outcrossed an $\mathrm{AB}^{\mathrm{mut}} / \mathrm{AB}$ fish (where mut represents the J101 mutation) with a WIK/WIK fish (a commonly used mapping strain) to generate $\mathrm{AB}^{\mathrm{mut}} / \mathrm{WIK}$ carriers. We incrossed the carriers to generate mapping panels, which are collections of embryos from individual pairs of fish. For high-resolution mapping, we genotyped 979 total embryos from four mapping panels. Because the commonly used markers were not informative, we designed new repeat markers to define the critical region. Linkage analysis of these embryos with polymorphic PCR products produced two nonoverlapping pools of recombinant embryos.

The new markers are as follows: BX323586.10-SSR1-F (close to Z20031) 5'-3' (GCCAGAGGTGAAAATGAGGA); BX323586.10-SSR1-R (AGATCCTGCTGGTCGTCTGT); BX323586.10-SSR2-F (close to Z20031) (TTGCAGAGAATACATGG); BX323586.10-SSR2-R (CAATGTGCATACTTTCA); BX247868.13-SSR2-F (close to Z26463) (TGGGCAGATTTATTGCAGTG); BX247868.13-SSR2-R (CTGCATCAAATTGCTTTTGG); Z26463-F (GGCGGAAGCTTTCTCTTTCT); Z26463-R (TTTTGGGTGAAATCCATTTG); ttna-SSR1-F (within the genomic boundaries of the ttna gene) (TTGTGGGACTCCAGGTTTCT); ttna-SSR1-R (AGTCCATTCGGTGAGTGTCA); ttna-exon38-SNP-F (within exon 38 of ttna) (TATCACCCGTAGCCTTGTCC); ttna-exon38-SNP-R (TCCCAGAGTCCGTTATTGCT); ttna-exon10-SNP-F (within exon 10 of ttna) (GTGCTGAAGCAAACAGGTGA); ttna-exon10-SNP-R (GAAAAGGAGGTGGAGGAACC); zC149F19-SSR1 (or CH211-149F19-SSR1)-F (CCCAGGAAGAATTTGTGTGTG); zC149F19-SSR1-R (TCCATAAGACTCATCAAATGCAC); z14949-F (GATGGGGGAAACAAAAAGGT); z149F19-R (GGAAAATCGCAGCTGAGTTC); AL713987.7-SSR2-F (close to Z14949) (ACCTAGGTCGCCTCTAT); AL713987.7-SSR2-R (TGGTTCTATGGATAAAC).

D-Tubocurarine treatments. Treatments were performed based on a previously described protocol (Liu and Westerfield, 1990). AB embryos were allowed to grow up to $\sim 21 \mathrm{hpf}$ in $\mathrm{E} 3$ embryo medium (in mM: 5 $\mathrm{NaCl}, 0.17 \mathrm{KCl}, 0.33 \mathrm{CaCl}_{2}, 0.33 \mathrm{MgSO}_{4}$ ), and then transferred to modified Barth's saline (MBS) with 4\% DMSO (Grinblat et al., 1999). We used dissecting needles to cut off the tip of the tail through at least one posterior somite, making sure not to pierce the yolk. We then transferred the embryos into a 24-well plate containing MBS and DMSO (vehicle) or vehicle with $60 \mu \mathrm{M}$ TC. Each well contained $1 \mathrm{ml}$ of liquid and $10 \mathrm{em}$ bryos, and the embryos were kept in this medium until fixation. The embryos were determined to be paralyzed if they did not display any spontaneous muscle contractions over the course of a $60 \mathrm{~s}$ observation, and did not display touch-evoked muscle contractions after being prodded three times. Any embryos in TC that were not paralyzed were discarded. Control embryos had their tails cut and were raised in vehicle medium and were indistinguishable from untreated controls grown in E3.

Chondroitinase $A B C$ treatments. $A B$ embryos at 21 hpf were transferred from E3 medium into MBS with 4\% DMSO, $60 \mathrm{~mm}$ sodium acetate, and $1 \mathrm{mg} / \mathrm{ml}$ bovine serum albumin. The tails were then cut, and the embryos were transferred to a 24-well plate containing the same MBS medium with chondroitinase ABC ( $2 \mathrm{U} / \mathrm{ml}$; Sigma-Aldrich) until fixation at 24 hpf. Control embryos, either cut or uncut, were raised in vehicle medium and were indistinguishable from untreated controls grown in E3.

Agarose embedding. At $19 \mathrm{hpf}$, unanesthetized AB embryos were mounted individually on a glass slide in $1 \%$ low-melting-point agarose in E3 with 15 mM HEPES. The agarose was allowed to set, and a small area of agarose was removed with a needle until just the tail was free. The slide containing the embryos was submerged in a $100 \times 25 \mathrm{~mm}$ Petri dish filled with E3. The embryos were removed from the agarose immediately before fixation. Any embryos that had escaped from the agarose or had reduced pigment compared with unmounted controls were discarded.

Immunohistochemistry and in situ hybridization. Digoxygenin-UTPlabeled riboprobes were synthesized by in vitro transcription and hydrolyzed to an average length of $\sim 300$ bases by limited alkaline hydrolysis (Cox et al., 1984). The probes were used at the following ages: crestin (18, 19, and $24 \mathrm{hpf}$ ), dct (2 dpf), ednrb1 (24 hpf), efna5a (24 hpf), epha4a (24 hpf), gch2 (30 hpf), gli1 (24 hpf), gli2 (24 hpf), isl1 (24 hpf), myod1 (11 and $24 \mathrm{hpf}$ ), ntn1a (24 hpf), pax2a (24 hpf), plxnala (24 hpf), plxnalb (24 hpf), plxna2 (24 hpf), plxna3 (24 hpf), plxna4 (24 hpf), sema3aa (24 hpf), sema3d (24 hpf), sema3 h (24 hpf), shha (24 hpf), slit1a (24 hpf), slit1b (24 hpf), slit2 (18, 24, and $28 \mathrm{hpf})$, slit3 (24 hpf), sox10 (19 and $24 \mathrm{hpf}$ ), and $x d h(30 \mathrm{hpf})$. Whole-mount in situ hybridization was performed as described previously (Halloran et al., 1999).

Whole-mount immunohistochemistry was performed as described previously (Wolman et al., 2004), unless otherwise specified. Briefly, embryos were fixed in $4 \%$ paraformaldehyde overnight, blocked in $5 \%$ sheep serum and $2 \mathrm{mg} / \mathrm{ml} \mathrm{BSA}$ in PBS, and incubated in primary antibody. The primary antibodies used were ZN-12 (1:500; recognizes HNK1), ZNP-1 (1:100; recognizes unknown antigen on motor axons), ZN-5 (1:500; recognizes Alcam) (all from Zebrafish International Resource Center, Eugene, OR), F59 (1:100; recognizes myosin heavy chain in slow muscle cells); F310 (1:200; recognizes myosin light chain 1 and $3 \mathrm{f}$ in fast muscle cells), 3A10 (1:50; recognizes neurofilament-associated antigen) (all from Developmental Studies Hybridoma Bank), anti-acetylated $\alpha$-tubulin (1:1500), anti-laminin (1:400) (both from Sigma-Aldrich), anti-collagen I (1:500; Santa Cruz Biotechnology), and anti-green fluorescent protein (GFP) (1:1000; Invitrogen). Antibodies against fibronectin (1:200), chondroitin sulfate proteoglycans (clone CS-56; 1:200) (both from Sigma-Aldrich), and tenascin (1:100; Millipore Bioscience Research Reagents) required additional washes through methanol and acetone as previously described (Zeller and Granato, 1999). Antibodies against desmin (1:100), $\alpha$-actinin (1:100), and titin (1:100) (all from Sigma-Aldrich) had shorter fixations and washes, as described previously (Costa et al., 2002). Antibody labeling was completed with the Vectastain $\mathrm{ABC}$ immunoperoxidase labeling kit (Vector Laboratories), or with Alexa 488- or Alexa 555-conjugated secondary antibodies ( $4 \mu \mathrm{g} / \mathrm{ml}$; Invitrogen).

Mosaic labeling. To mosaically label individual RB axon arbors, we microinjected embryos at the one to four cell stage with DNA constructs that drive GFP expression in RB neurons ( $25 \mathrm{ng} / \mu \mathrm{l}$; $0.2 \mathrm{nl})$. We used either the -3.1ngn1:gfp construct (Blader et al., 2003) (provided by Uwe Strähle, Karlsruhe Institute of Technology, Karlsruhe, Germany) or the SSX:gfp construct (Uemura et al., 2005) (provided by Hitoshi Okamoto, RIKEN Brain Science Institute, Saitama, Japan). The embryos were fixed at $28 \mathrm{hpf}$ and labeled with immunohistochemistry against GFP. We only selected neurons for analysis that were clearly isolated and did not have arbors that overlapped with axons from other labeled neurons.

Time-lapse imaging. 1,1'-Dioctadecyl-3,3,3',3'-tetramethylindocarbocyanine perchlorate (DiI) injections were performed as described previously (Liu and Halloran, 2005). Embryos were anesthetized in $0.02 \%$ 3 -amino benzoic acid ethylester (tricaine) and immobilized in 3\% methyl cellulose. A $0.2 \%$ solution of DiI (Invitrogen) was pressureinjected into one to three localized sites along the axis of the dorsal spinal 
cord. Embryos were anesthetized and mounted starting $\sim 21 \mathrm{hpf}$, well after the time when muscles begin contracting in WT embryos, and were imaged for 2-10 h. For imaging, the embryos were mounted in $1 \%$ lowmelting agarose in E3 with $0.02 \%$ tricaine and 15 mM HEPES. Imaging was performed at $27-28^{\circ} \mathrm{C}$. Images were captured on a Nikon E-600FN equipped with standard epifluorescence, a filter wheel, and a CoolSnap HQ camera (Photometrics). Images were captured every $1 \mathrm{~min}$, and exposure times were typically $100-600 \mathrm{~ms}$.

Acridine orange staining. Live $24 \mathrm{hpf}$ embryos were dechorionated and incubated in acridine orange $(5 \mu \mathrm{g} / \mathrm{ml}$ in E3; 3,6-bis[dimethylamino]acridine hemi-zinc chloride; Sigma-Aldrich) for $30 \mathrm{~min}$. The embryos were then washed, mounted on coverslips, and visualized.

Fixed embryo imaging. All bright-field images were captured on a Nikon TE300 inverted microscope equipped with a Spot RT camera (Diagnostic Instruments) and processed with MetaMorph software (Molecular Devices). Fluorescent images are confocal projections captured on an Olympus Fluoview1000 on an IX81 microscope or a Bio-Rad MRC 1024 on a Zeiss Axiovert microscope.

Quantifications. For quantification of axon arbor morphology, images from laterally mounted embryos were aligned with the central RB axons along the $90^{\circ}$ axis, making ventral equal $0^{\circ}$ (see Fig. $4 A$ ). The initial angle of each axon (at least $10 \mu \mathrm{m}$ long) was measured from a branch point toward the growth cone, averaged over a length of $10 \mu \mathrm{m}$. The extent of the axon arbors along the anterior-posterior (A-P) body axis were measured as the distance from the anterior-most to the posterior-most points of the arbors along an axis parallel to the central $\mathrm{RB}$ axons. The ventral (V)-to-longitudinal (L) and L-to-V measurements were done by comparing the initial angle of each axon from a branch point to the final angle of the axon immediately before it branched again or ended in a growth cone. Angles of $0-45^{\circ}$ were designated as ventral, $46-135^{\circ}$ as longitudinal, and $136-180^{\circ}$ as dorsal. The number of branches per axon arbor was determined by counting the number of axon termini.

For the time-lapse imaging experiments, the axon behaviors were only quantified if both axons were in focus and could be followed for at least 5 min before and after an encounter. Growth rates were quantified if axons could be reliably followed for at least $10 \mathrm{~min}$, and were calculated as the total distance traveled divided by time. Landmarks on the embryo were used to compensate for drift during imaging.

Images were processed and analyzed with MetaMorph software (Molecular Devices). The graphs were constructed with Microsoft Excel software. The initial growth, extent of the axon arbors along the A-P axis, and growth rate data are reported as mean \pm SEM. Statistical significance was assessed by Student's $t$ test. The data from the V to L, L to V, or axon behaviors are reported as percentage of the total in each group. Statistical significance was assessed by Fisher's exact test using the GraphPad Software QuickCalcs Online Calculator for Scientists (GraphPad Software).

\section{Results}

\section{Characterization of titin $\boldsymbol{a}(\text { pik) })^{u w 2}$}

We isolated a mutant with defects in heart and skeletal muscle contractions, initially designated J101, which is similar to a previously identified allele of the titin a (tna) mutant pickwick (pik) $^{m V O 62 H}$ (Xu et al., 2002). We mapped the J101 mutation to a region on chromosome 9 (supplemental Fig. 1, available at www. jneurosci.org as supplemental material) defined on one side by the newly designed repeat markers BX247868.13 SSR-2 (near z26463; 6 recombinants/979 mutants) and on the other side by zC149F19-SSR1 ( 6 recombinants/979 mutants). Because the ttna gene had previously been mapped to this region (Xu et al., 2002; Sato and Mishina, 2003; Seeley et al., 2007; Steffen et al., 2007), we identified new markers within the ttna gene. Markers in exons 89-90 (ttna-SSR1) and exon 38 (ttna-exon38-SNP) each had 1 recombinant of 979 mutants tested, and a marker in ttna exon 10 (tna-exon10-SNP) had zero recombinants. In addition, we made complementation crosses between carriers of this mutation and carriers of ttna (pik) $)^{m 171}$ (Xu et al., 2002) and found that $25 \%$ of the offspring displayed the ttna phenotype of defective heart contractions. The phenotypic similarities to other tna alleles, the failure to complement ttna (pik) ${ }^{m 171}$, and the mapping data together indicate that the J101 mutation is likely an allele of ttna (pik), which we named titin a (pik) $)^{u w 2}$, and will refer to as ttna ( $p i k$ ) for the remainder of this paper. This mutation is recessive, and the phenotype has been stable and consistent throughout five generations.

ttna (pik) mutant embryos appear morphologically grossly normal at $24 \mathrm{hpf}$ (data not shown). However, the embryos exhibit very limited spontaneous and touch-stimulated muscle contractions up to $\sim 28 \mathrm{hpf}$. There are many fewer spontaneous contractions than in WT embryos, and ttna (pik) embryos only bend slightly without forming full C-bends (supplemental Movie 1, available at www.jneurosci.org as supplemental material). As the somites mature, the muscles progressively stop contracting, so the contractions are limited to the youngest, most posterior muscles. After $28 \mathrm{hpf}$, skeletal muscle contraction stops entirely. The cardiac muscles show similar defects in contraction. At 2 and 3 $\mathrm{dpf}$, the mutant embryos develop pericardial edema (Fig. $1 B, D$, arrows), and the somites develop a grayish appearance, which may be indicative of cell death (Fig. $1 D$, arrowhead), although there is no increase in apoptosis based on acridine orange staining (data not shown). Moreover, normal muscle striation is not visible in ttna (pik) embryos (Fig. $1 E, F$ ). At even later ages, the tna ( $p i k$ ) mutant embryos develop edema around the yolk sac and the eyes (data not shown). They survive until 7-10 $\mathrm{dpf}$, which is similar to other mutants that affect heart contraction and circulation.

Antibody staining for the myosin heavy chain in slow muscle cells shows nicely organized fibrils in the WT embryos (Fig. $1 G$, arrows). In ttna (pik) embryos, this staining is still present in the correct area of the somite but is highly disorganized (Fig. $1 \mathrm{H}$, arrows). A similar disruption in staining is also seen with antibodies against the myosin light chain in fast muscle cells, desmin, $\alpha$-actinin, and titin (data not shown). This disruption in sarcomere proteins has been shown in other mutations or knockdowns of titin (Seeley et al., 2007; Steffen et al., 2007). Although the sarcomeres are disrupted, the specific myosin staining shows the slow and fast muscle fiber types seem to be present and in the correct positions of the somite.

\section{ttna (pik) mutant embryos have defects in peripheral Rohon-Beard axon guidance}

Interestingly, we found defects in the sensory RB axons that extend over the somites. RB cells are mechanosensory neurons, whose cell bodies reside in two bilateral rows in the dorsal spinal cord and extend central axons longitudinally in the spinal cord (Clarke et al., 1984; Bernhardt et al., 1990). Each RB also has a peripheral axon that extends orthogonally out of the dorsal spinal cord and then grows in a general ventral direction between the somite and epidermis to innervate the epidermis of the trunk (Fig. 2 A,C) (Bernhardt et al., 1990; Kuwada et al., 1990; Metcalfe et al., 1990). In the ttna (pik) embryos, some of the peripheral RB axons grow in a longitudinal direction, instead of the normal ventral direction (Fig. $2 B, D$, arrow). These defects were also seen at $21 \mathrm{hpf}$ when the peripheral axons have only begun to grow out (data not shown). In the ttna (pik) ${ }^{m 171}$ allele, which has only cardiac muscle defects, the peripheral RB axons appear normal (data not shown). 


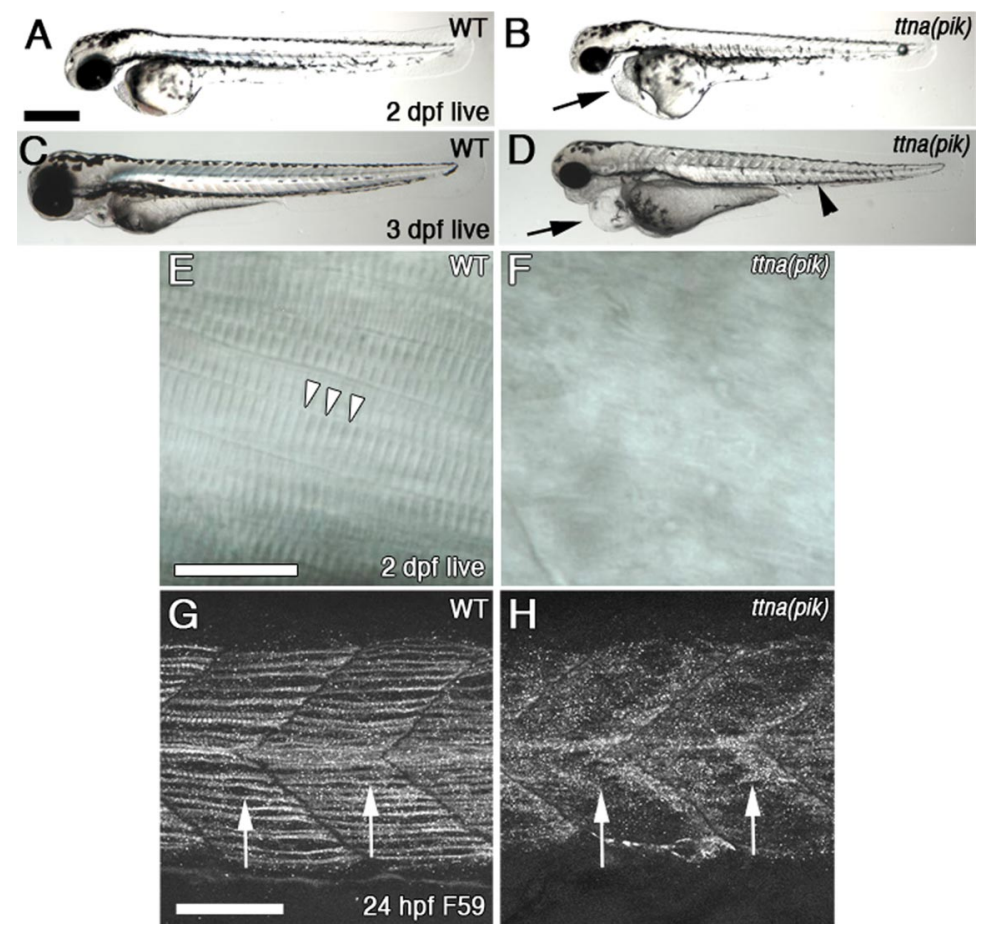

Figure 1. ttha (pik) embryos develop edema and muscle defects. Anterior is to the left. $A-D$, Differential interference contrast (DIC) images of liveWT $(\boldsymbol{A}, \boldsymbol{C}$ and ttna (pik) $(\boldsymbol{C}, \boldsymbol{D})$ embryos at $2 \mathrm{dpf}(\boldsymbol{A}, \boldsymbol{B})$ and $3 \mathrm{dpf}(\boldsymbol{C}, \boldsymbol{D})$. The ttha (pik) embryos develop pericardial edema $(\boldsymbol{B}, \boldsymbol{D}$, arrow) and a grayish appearance to their muscles ( $\boldsymbol{D}$, arrowhead). $\boldsymbol{E}, \boldsymbol{F}$, DIC images of lateral mounts of $2 \mathrm{dpf}$ embryos. $\boldsymbol{E}$, In WT embryos, muscle striations are visible, indicating segmental organization of the sarcomeres (arrowheads). $\boldsymbol{F}$, In ttna (pik) muscle tissue, the striations are absent. $\mathbf{G}, \boldsymbol{H}$, Lateral views of anterior trunk at $22 \mathrm{hpf}$ showing $\mathrm{F} 59$ antibody labeling of slow muscle myosin. $\boldsymbol{G}$, Myosin is organized into longitudinal fibers in WT embryos (arrows). $\boldsymbol{H}$, In ttha (pik) embryos, myosin is still present in the somites, but it has a disorganized distribution (arrows). Scale bars: $\boldsymbol{A}-\boldsymbol{D}, 400 \mu \mathrm{m} ; \boldsymbol{E}, \boldsymbol{F}, 20 \mu \mathrm{m} ; \boldsymbol{G}, \boldsymbol{H}, 40 \mu \mathrm{m}$.

Other axons, migratory cells, and guidance cue expression are normal in ttna (pik) mutants

We also examined other axons and migratory cell types to determine whether their guidance is disrupted in the ttna (pik) mutants. We were particularly interested in the spinal motor axons and trunk neural crest cells because they have been shown to require muscle-derived cues for correct guidance and migration (Halloran and Berndt, 2003; Young et al., 2004; Feldner et al., 2005; Honjo and Eisen, 2005; Schweitzer et al., 2005; Sato-Maeda et al., 2006; Schneider and Granato, 2006). Both the spinal motor axons and neural crest cells showed normal development in ttna (pik) embryos (Fig. $2 E-H$, arrows). In addition, we examined several other axon tracts throughout the nervous system, including the hindbrain reticulospinal axons, the dorsal longitudinal fasciculus, medial longitudinal fasciculus, anterior commissure, posterior commissure, tract of the postoptic commissure, retinal ganglion cell axons, epiphysial axons, posterior lateral line axons, and branchiomotor axons. We did not find defects in any of these axon pathways in ttna (pik) embryos (data not shown). These results suggest that the cue disrupted in tna (pik) embryos is only necessary for peripheral RB axon guidance, and not the guidance of these other cell types.

To ask whether the expression of axon guidance cues or receptors is disrupted in ttna (pik) mutants, we performed in situ hybridization for a number of such molecules that are expressed in the vicinity of the muscles or RB cells. These included ephrin-a5a, Eph-a4a, netrin-1a, plexin-a1a, plexin-a1b, plexin-a2, plexin-a3, plexin-a4, semaphorin3aa, semaphorin3d, semaphorin $3 h$, shha, slit1a, slit1b, slit2, and slit3. We also performed antibody labeling for components of the extracellular matrix that are normally present in the area in which the peripheral RB axons grow, including laminin, collagen I, fibronectin, tenascin, and chondroitin sulfate proteoglycans (CSPGs). The expression of these molecules appeared normal in ttna (pik) embryos (data not shown), suggesting that the defective muscle does not act by altering the expression patterns of these guidance cues.

\section{Other muscle mutants also have defects} in the peripheral $\mathrm{RB}$ axons

Muscle cells are the only cells that express titin a (Xu et al., 2002), suggesting they are the source of the RB axon guidance cue. To further test the role of muscle development or function in RB axon guidance, we examined three mutants in the sonic hedgehog signaling pathway, which is required for proper muscle development. sonic hedgehog [shha (syu)] mutant embryos lack muscle pioneer cells, which are the first muscle cells to differentiate and contract, beginning 17 hpf (Hatta et al., 1991). The other muscle cells begin contracting $\sim 19$ hpf (Hatta et al., 1991). shha (syu) mutants also have a reduction in slow muscle cells, and some defects in the guidance of the primary motor axons (Brand et al., 1996; van Eeden et al., 1996; Schauerte et al., 1998; Lewis et al., 1999), which stimulate the muscles to contract by releasing acetylcholine (ACh) (Myers et al., 1986; Hanneman and Westerfield, 1989; Westerfield et al., 1990). Consequently, shha (syu) embryos do not have muscle contractions before 19 hpf, but do show contractions by $21 \mathrm{hpf}$ (our unpublished observations). The gli2a (yot) and smoothened [smo (smu)] mutant embryos lack slow muscle cells and muscle pioneer cells, and have severe defects in primary motor axons (Brand et al., 1996; van Eeden et al., 1996; Karlstrom et al., 1999; Varga et al., 2001). They have no muscle contractions before $24 \mathrm{hpf}$ (our unpublished observations). We found that the peripheral RB axons in all of these mutants have longitudinally directed axons (Fig. $3 B-D$, arrows), similar to the tna ( $p i k$ ) embryos. These results further support the idea that normal muscle development and function is required for $\mathrm{RB}$ axon guidance.

As a control, we examined lamal (bal) embryos, which have convergent/extension defects resulting in a shorter body axis and altered gross somite morphology, but the muscle types, primary motor neurons, and muscle contractions all appear normal (Karlstrom et al., 1996; Stemple et al., 1996; Paulus and Halloran, 2006; Pollard et al., 2006). Because the body axis is shorter in lama1 (bal) mutant embryos, the $\mathrm{RB}$ neurons and axons are denser (Paulus and Halloran, 2006), but the peripheral axons extend in the normal ventral direction (Fig. $3 E$ ).

Individual RB axon arbors extend significantly farther along the A-P body axis and show increased longitudinal growth in muscle mutants

To further characterize and to quantify the RB axon guidance defects, we mosaically labeled small numbers of RB neurons by 

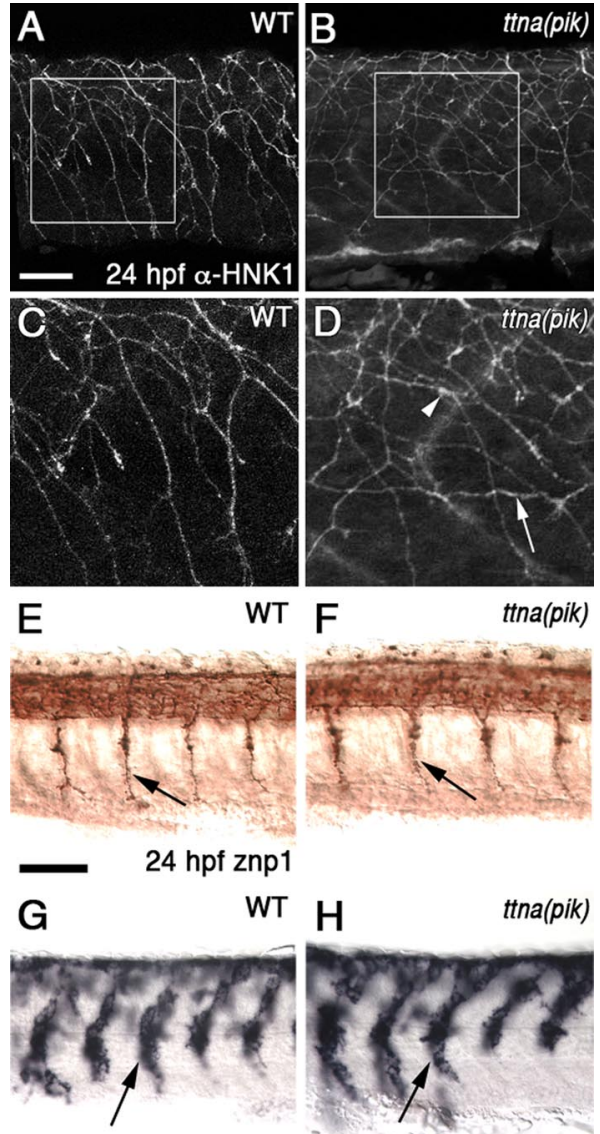

24 hpf crestin

Figure 2. ttna (pik) mutants have defects in peripheral RB axons, whereas motor axons and neural crest cells migrate normally. $\boldsymbol{A}-\boldsymbol{D}$, Lateral views of embryos at $24 \mathrm{hpf}$ showing RB axons labeled with $\alpha$-HNK1. A, C, Peripheral RB axons in WT embryos generally grow ventrally and avoid each other. $\boldsymbol{C}$ is a higher magnification view of the boxed area in $\boldsymbol{A} . \boldsymbol{B}, \boldsymbol{D}$, In tha (pik) embryos, some axons grow longitudinally (arrows) or appear to make extended contacts with each other (arrowheads). $\boldsymbol{D}$ is a higher magnification view of the boxed area in $\boldsymbol{B}$. $\boldsymbol{E}, \boldsymbol{F}$, Lateral views of primary spinal motor axons labeled with znp1 (arrows). Axons extend normally in both WT (E) and ttna (pik) (F) embryos. $\mathbf{G}, \boldsymbol{H}$, Lateral views of anterior trunk at $24 \mathrm{hpf}$ showing crestin in situ hybridization in neural crest cells (arrows), which appear to migrate normally in both WT (G) and ttna (pik) $(\boldsymbol{H})$ embryos. Scale bars: $\boldsymbol{A}-\boldsymbol{D}, 10 \mu \mathrm{m} ; \boldsymbol{E}-\boldsymbol{H}, 60 \mu \mathrm{m}$.

injecting embryos with a DNA construct that drives GFP in the RB neurons (see Materials and Methods). This method allows us to visualize arbors of individual, isolated RB neurons without obstruction by other labeled cells. In WT embryos, individual axon arbors extend ventrally and are generally restricted in their longitudinal direction to cover approximately two somites (Fig. $4 A-C)$. In contrast, arbors in the muscle mutant embryos extended significantly farther along the A-P body axis, with some axons extending longitudinally over many somites (Fig. $4 D-F$ ). We measured the A-P extent of the axon arbors and found that arbors in WT embryos had an average A-P distance of $161.9 \pm$ $11.4 \mu \mathrm{m}(n=20$ arbors, 19 embryos) (Fig. $4 G)$. The gli2a (yot) embryos, which have no muscle contractions, had arbors that extended significantly farther along the A-P axis than WT $(281.9 \pm 28.9 \mu \mathrm{m} ; n=16$ arbors, 15 embryos; $p=0.001)$ (Fig. $4 D-F)$. The embryos with only a partial loss of muscle contractions were less severely affected. The A-P arbor extent was still significantly greater than WT in shha (syu) embryos $(234.5 \pm$ $25.6 \mu \mathrm{m} ; n=20$ arbors, 17 embryos; $p=0.02)$, whereas arbors in the ttna (pik) (179.1 $\pm 14.3 \mu \mathrm{m} ; n=23$ arbors, 22 embryos; $p=0.35)$ and lama1 (bal) $(141.3 \pm 13.0 \mu \mathrm{m} ; n=21$ arbors, 20
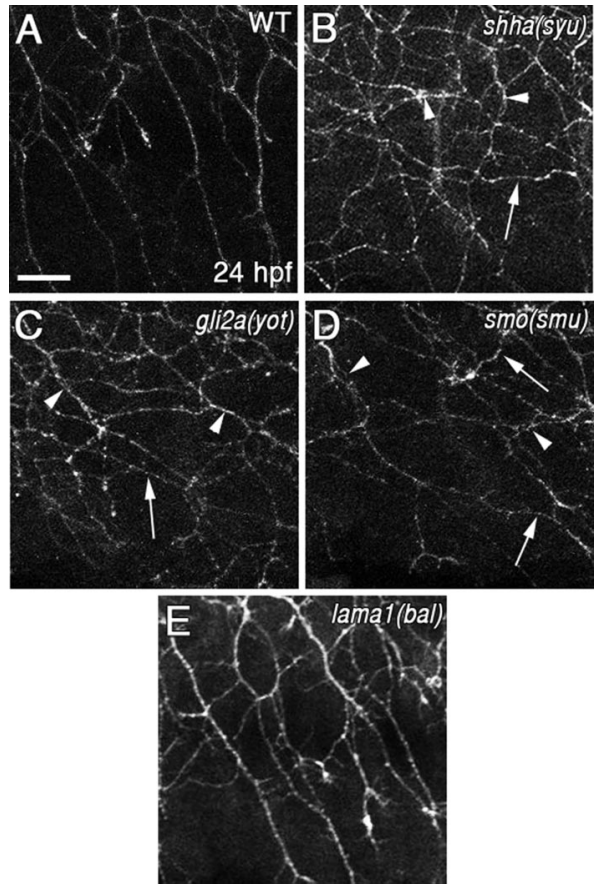

Figure 3. Muscle mutants have peripheral RB axon guidance defects. $\boldsymbol{A}-\boldsymbol{E}$, Lateral views of embryos at $24 \mathrm{hpf}$ showing RB axons labeled with $\alpha$-HNK1. $\boldsymbol{A}$, Peripheral RB axons in WT embryos generally grow ventrally and avoid each other. $\boldsymbol{B}-\boldsymbol{D}$, In shha (syu) (B), gliza (yot) (C), and smo (smu) (D) mutant embryos, some axons grow longitudinally (arrows) or appear to make extended contacts with each other (arrowheads). $\boldsymbol{E}$, The peripheral RB axons in lama1 (bal) embryos appear to be like WT. Scale bar, $20 \mu \mathrm{m}$.

embryos; $p=0.24)$ mutants were not significantly different from WT.

As another assessment of longitudinal growth, we measured the initial direction of growth of each axon from a branch point (ventral is $0^{\circ}$; dorsal is $180^{\circ}$ ) (Fig. $4 A$ ) and saw that embryos with muscle defects had a larger (more longitudinal) angle of growth (Fig. $4 H$ ). The axons in WT embryos grew at an average initial angle of $55.3 \pm 1.9^{\circ}(n=583$ axons). The axons in tna (pik) $\left(61.9 \pm 1.5^{\circ} ; n=866\right.$ axons; $\left.p=0.006\right)$, shha (syu) $\left(61.7 \pm 1.4^{\circ}\right.$; $n=891$ axons; $p=0.007)$, and gli $2 a$ (yot) $\left(64.1 \pm 1.7^{\circ} ; n=655\right.$ axons; $p=0.0006$ ) embryos were all significantly more longitudinal (larger angle). The lama1 (bal) embryos, which have normal muscle contractions, were not significantly different from WT $\left(58.0 \pm 1.8^{\circ} ; n=692\right.$ axons; $\left.p=0.30\right)$.

To determine why the ttna (pik) mutants initially extend axons more longitudinally but the A-P arbor length was not larger, we examined the percentage of axons that changed direction throughout their path. First, we quantified the percentage of axons that began growing ventrally (angle of $0-45^{\circ}$ ) but then turned to end up more longitudinal $\left(46-135^{\circ}\right)$ (V to L) (Fig. 4I). In WT embryos, the $\mathrm{V}$ to L was only $6 \%$ ( $n=259$ ventral axons), suggesting that the majority of ventral axons remain ventral instead of turning longitudinally. Most of the embryos with muscle contraction defects had a much higher V-to-L percentage. The gli2a (yot) embryos had the largest difference from WT $(20 \% ; n=$ $197 ; p=0.0001)$. Once again, the mutants that had some muscle contractions, ttna (pik) (13\%; $n=298 ; p=0.01)$ and shha (syu) $(18 \% ; p=0.0001)$ were less severely affected, although still different from WT. The lamal (bal) embryos also showed a higher V-to-L percentage $(12 \% ; n=387 ; p=0.01)$, which may be attributable to the axons being denser, and therefore more likely to encounter other axons and turn. 

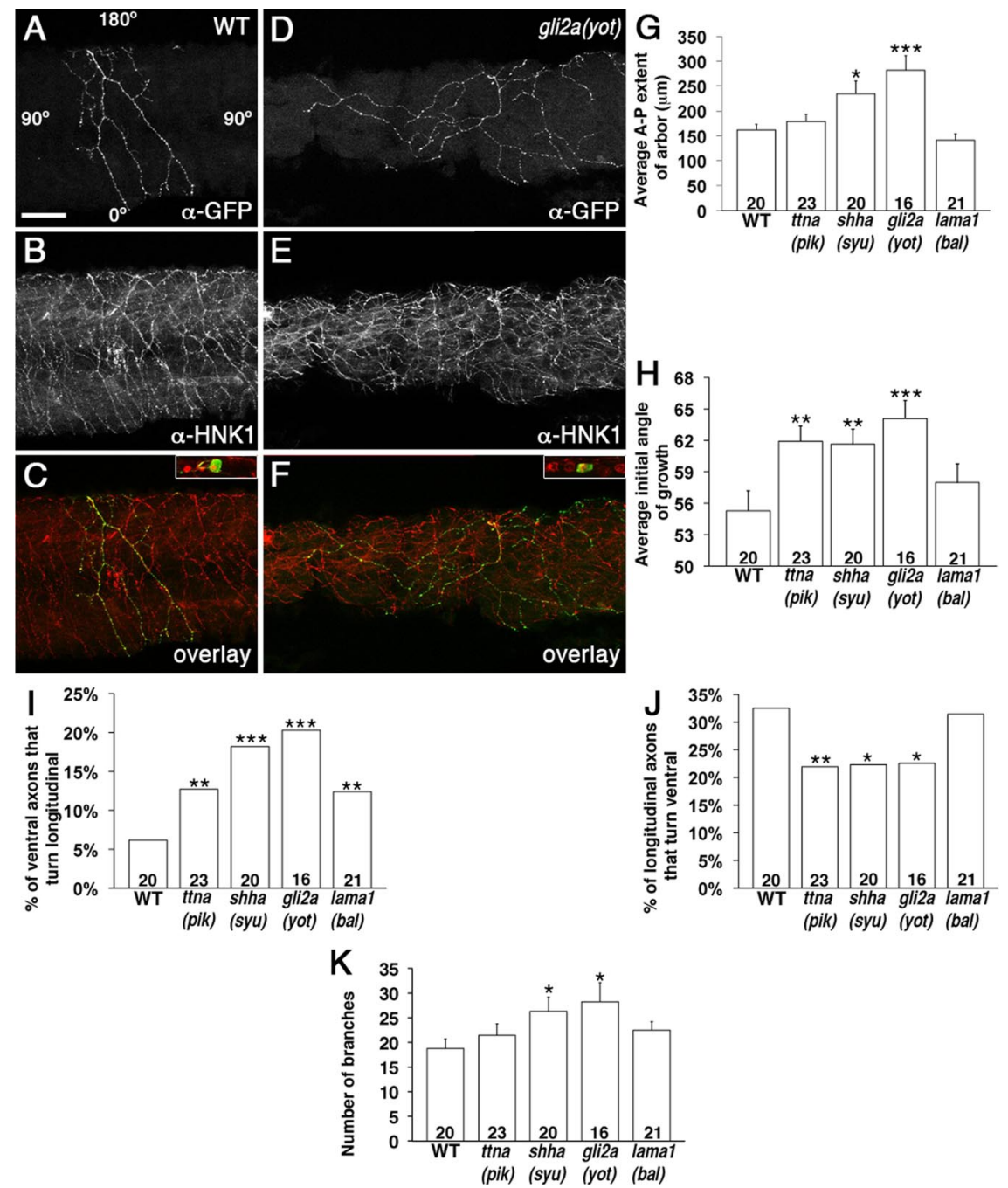

Figure 4. Peripheral $\mathrm{RB}$ axon arbors in mutant embryos show defects in arbor size and direction of growth. $\mathbf{A}-\boldsymbol{F}$, Lateral views with anterior to the left of embryos with single RB neurons labeled with $\alpha$-GFP $(\boldsymbol{A}, \boldsymbol{D})$, and all RB axons labeled with $\alpha$-HNK1 $(\boldsymbol{B}, \boldsymbol{E}) . \boldsymbol{C}$ and $\boldsymbol{F}$ are overlays of $\alpha$-GFP and $\alpha$-HNK1 labeling. The insets show focal plane of cell bodies, indicating multiple cells labeled with $\alpha$-HNK-1 (red), but only one colabeled with $\alpha$-GFP (green). $A-C$, Representative peripheral axon arbor in WT embryo showing axons grow in a general ventral (down) direction and are restricted along the anterior-posterior axis. Angle labels in $\boldsymbol{A}$ show the axes used to measure the axon angles. $\boldsymbol{D}-\boldsymbol{F}$, Representative arbor in a gliza (yot) embryo showing axons grow more longitudinally and arborize over a greater anterior-posterior distance. Scale bar, $10 \mu \mathrm{m}$. G-K, Quantification of A-P extent of arbor $(\boldsymbol{G})$, initial growth angle of axons $(\boldsymbol{H})$, percentage of axons that initially grow ventral but turn longitudinal $(\boldsymbol{I})$, percentage of axons that initially grow longitudinal but turn ventral $(\boldsymbol{J})$, and the number of axon branches $(\boldsymbol{K}){ }^{*} p<0.05,{ }^{* *} p<0.01,{ }^{* * *} p<0.005$. Numbers on graphs indicate number of axon arbors. Error bars indicate SEM.

Similarly, we measured the percentage of axons that began longitudinally and ended up turning more ventrally (L to V) (Fig. $4 J)$. In WT embryos, this was quite high (33\%; $n=166$ longitudinal axons), suggesting many longitudinal axons corrected their direction to grow ventral, and probably thus limit the extent of the arbor along the A-P axis. The gli2a (yot) $(23 \% ; n=266 ; p=$ $0.03)$, tha (pik) (22\%; $n=369 ; p=0.01)$, and shha (syu) $(22 \%$; $n=354 ; p=0.02$ ) all had lower L-to-V percentages than WT, whereas the lamal (bal) $(31 \% ; n=318 ; p=0.84)$ were not significantly different. On the whole, these data suggest that axons in the muscle mutants have a greater propensity to grow in the longitudinal direction.

Finally, we quantified the number of axon branches (see Materials and Methods) in the embryos to see whether that was also affected (Fig. $4 K$ ). The WT embryos had an average number of $18.8 \pm 1.9$ branches per axon arbor. The gli2a (yot) $(28.3 \pm 3.9$; $p=0.04)$ and shha (syu) $(26.3 \pm 2.9 ; p=$ $0.04)$ embryos had significantly more branches than WT. The ttna (pik) (21.4 \pm $2.3 ; p=0.38)$ and lamal (bal) $(22.5 \pm 1.7$; $p=0.16$ ) embryos were not significantly different. This increase in branch number may be caused by an increase in the formation of branches or by a decrease in their elimination.

Muscle mutants have defects in contact repulsion between peripheral $\mathrm{RB}$ axons The peripheral RB axons in WT embryos repel one another on contact (Liu and Halloran, 2005; Sagasti et al., 2005), which likely serves to maximize the innervated surface area and to restrict individual arbors to specific nonoverlapping regions. The mutants with defects in muscle contraction all have axons that grow more longitudinally, suggesting these axons may have reduced mutual repulsion. In our analysis of fixed, antibody-labeled embryos, we saw that WT axons do not appear to make extensive contact with each other (Figs. $2 A, C, 3 A$ ), whereas axons in the muscle mutants at times appear to make extended contacts (Figs. $2 B, D, 3 B-D$, arrowheads). To test whether muscle mutants indeed have defects in contact repulsion, we performed time-lapse imaging of WT, ttna (pik), and shha (syu) embryos and analyzed behaviors of RB axons on contact.

In WT embryos (Fig. 5A-D, arrows; supplemental Movie 2, available at www. jneurosci.org as supplemental material), the majority of interactions between axons resulted in repulsion $(96 \% ; n=50$ interactions in 3 embryos), whereas only $4 \%$ of the interactions resulted in crossing over the other axon (Fig. 5I). In both ttna (pik) and shha (syu) mutant embryos, we often saw one axon growing along another axon for short distances before eventually retracting or crossing over (Fig. $5 E-H$, arrows; supplemental Movie 3, available at www.jneurosci.org as supplemental material). These mutants showed significantly fewer repulsive interactions. In ttna (pik) embryos, $68 \%$ of interactions $(n=28$ axons in 5 embryos; $p=0.001)$ resulted in repulsion and $32 \%$ in crossing over. In shha (syu) mutants, $66 \%$ of interactions $(n=32$ interactions in 3 embryos; $p=0.0004)$ resulted in repulsion and $34 \%$ in crossing over. Thus, reduced repulsion and increased ability to cross other axons likely contributes to the increased longitudinal growth in the muscle mutants.

Interestingly, we also noticed differences in the growth rates of the axons from the different groups of embryos (Fig. 5J). In the WT embryos, the axons traveled at an average rate of $14.7 \pm 1.5$ $\mu \mathrm{m} / \mathrm{h}$ ( $n=26$ axons in 6 embryos). Axons grew faster in both ttna (pik) (24.7 $\pm 1.8 \mu \mathrm{m} / \mathrm{h} ; n=37$ axons in 7 embryos) and sha (syu) embryos ( $24.3 \pm 1.8 \mu \mathrm{m} / \mathrm{h} ; n=37$ axons in 3 embryos; $p=$ 0.000009 ). These data suggest that reduced repulsion also allows axons to extend more rapidly. 


\section{Blocking nerve stimulation of the muscle prevents muscle contractions and causes peripheral RB axon guidance defects}

Our analysis of muscle development mutants shows a general correlation between the severity of peripheral RB axon guidance defects and the degree of muscle contraction loss. Moreover, in both zebrafish and Xenopus laevis embryos, the peripheral $\mathrm{RB}$ axons begin growing out at the same time as muscle contractions begin ( $17 \mathrm{hpf}$ in zebrafish, stage 22 in Xenopus), supporting the possibility that these two processes may be related (Muntz, 1975; Myers et al., 1986; Metcalfe et al., 1990; Somasekhar and Nordlander, 1997). To test more directly whether muscle contractions themselves are guiding the peripheral axons, we used several approaches to specifically block contractions. We first treated embryos with the active ingredient of curare, TC, which is an irreversible competitive antagonist for nicotinic ACh receptors (nAChRs). TC treatment has previously been used to block early spontaneous muscle contractions in zebrafish and does not affect the guidance of the primary spinal motor axons (Grunwald et al., 1988; Liu and Westerfield, 1990). TC binds with high affinity at the interface between the $\alpha$ and $\gamma$ subunits of nAChRs and with low affinity at the interface of the $\alpha$ and $\delta$ sub-

units (Pedersen and Cohen, 1990). Between 18 and $24 \mathrm{hpf}$ in zebrafish, the somites are the only tissues expressing these combinations of receptors (Sepich et al., 1998; Zirger et al., 2003; Etard et al., 2005; Ackerman et al., 2009). We found that TC treatment completely blocked muscle contractions and also caused longitudinal growth of peripheral RB axons (Fig. $6 B$, arrows), supporting the idea that muscle contraction is required for $\mathrm{RB}$ ventral growth.

To test this idea further, we analyzed nicotinic acetylcholine receptor $\alpha 1$ [chrnal (nic-1)] mutants, which lack the $\alpha$ subunit to which TC binds (Sepich et al., 1998). The chrnal protein is only expressed in muscles, and these mutants have previously been shown to have a loss of muscle contractions but have normal primary motor axon pathfinding, even though they lack nAChRs (Westerfield et al., 1990). We found that these mutants, like the other muscle mutants, also showed longitudinal growth of peripheral $\mathrm{RB}$ axons (Fig. 6C, arrows). We quantified individual axon arbor parameters in chrnal (nic-1) mutants and found that their A-P extent and growth angle are significantly larger than WT (Fig. $6 E-I)$. The A-P extent is $215.0 \pm 19.9 \mu \mathrm{m}(n=23$ arbors, 21 embryos; $p=0.04$, compared with WT) and the axons initially grow at an average angle of $63.6 \pm 1.4^{\circ}(n=977$ axons; $p=0.0005$, compared with WT). They also have a significantly larger V-to-L percentage $(17 \% ; n=447$ ventral axons; $p=$ $0.0001)$, smaller L-to-V percentage $(18 \% ; n=508 ; p=0.0001)$ and larger number of branches $(25.3 \pm 2.5 ; p=0.04)$.

We also performed time-lapse imaging experiments on the chrnal (nic-1) mutants and found that they have significantly fewer repulsive interactions than WT $(41 \% ; n=34$ interactions in 6 embryos; $p=0.00000002$ ) (Fig. $6 J$ ) and faster axonal growth rates $(23.1 \pm 1.9 \mu \mathrm{m} / \mathrm{h} ; n=41$ axons in 6 embryos; $p<0.005)$.
Together, these data suggest that muscle contractions themselves are specifically causing the RB axon guidance defects.

\section{Physically blocking embryo movements causes peripheral RB axon defects}

Because all of the previous mutants or treatments affected nerve stimulation, muscle development, or intracellular muscle molecules, we sought a method to restrict only the large embryo movements resulting from muscle contractions, while minimizing effects on other physiological processes, such as secretion of potential guidance cues. To test the hypothesis that the mechanical force produced by muscle contractions is necessary to correctly guide the peripheral RB axons, we embedded the embryos in low-melting-point agarose to prevent the embryos from moving. We removed the agarose from the tips of the tails to provide room for the trunks of the embryos to extend normally. While embedded, the embryos are still capable of contracting their muscle cells, but the trunks are prevented from bending to the same degree as unembedded embryos. The peripheral RB axons that grow over the movement-restricted muscles show similar defects as those seen in the muscle mutants and TC-treated embryos, with axons growing longitudinally instead of ventrally (Fig. $6 D$, arrows) and the individual axon arbors extending significantly farther along the A-P axis and in a more longitudinal angle than WT or control embryos. The average A-P extent of the axon arbors is $226.2 \pm 19.0 \mu \mathrm{m}(n=22$ arbors, 20 embryos; $p=0.006)$ (Fig. $6 E$ ), the axons grow at an average initial angle of $67.5 \pm$ $21.5^{\circ}(n=1020$ axons; $p=0.0000004)$ (Fig. $\left.6 F\right)$, the $\mathrm{V}$ to $\mathrm{L}$ is $26 \%$ ( $n=406$ ventral axons; $p=0.0001$ ) (Fig. $6 G$ ), the $\mathrm{L}$ to $\mathrm{V}$ is $22 \%$ $(n=495$ longitudinal axons; $p=0.009)($ Fig. $6 H)$, and they have 

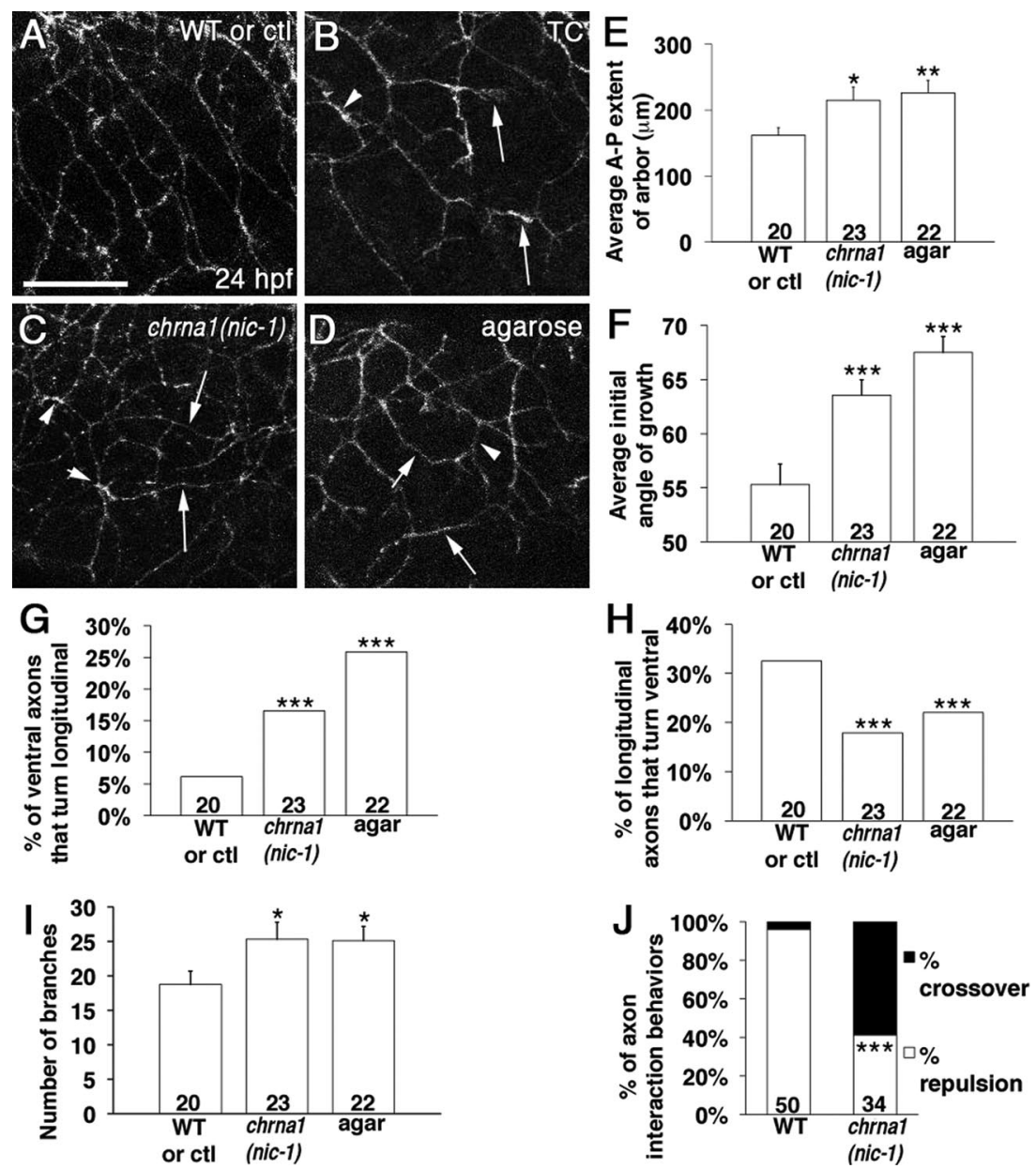

Figure 6. Blocking muscle contractions causes peripheral RB axon guidance defects. $\boldsymbol{A}-\boldsymbol{D}$, Lateral views of embryos at $24 \mathrm{hpf}$ showing RB axons labeled with $\alpha$-HNK1. $\boldsymbol{A}$, Peripheral RB axons in WT or control treated embryos generally grow ventrally and avoid each other. $\boldsymbol{B}$, Blocking muscle contraction in WT embryos by treating them with TC causes similar defects in the peripheral $\mathrm{RB}$ axons as those in the mutants that affect muscle contractions. Axons grow longitudinally (arrows) and make apparent extended contacts with each other (arrowheads). C, chrna1 (nic-1) embryos show similar defects with longitudinal axons (arrows) and axons making extended contact (arrowhead). D, Embedding WT embryos in agarose (agar) to limit muscle movements results in similar defects in the peripheral RB axons, with longitudinal axons (arrows) and axons making extended contacts (arrowheads). Scale bar, $20 \mu \mathrm{m}$. $\boldsymbol{E}-\boldsymbol{J}$, Quantification of individual peripheral RB axon arbors showing defects in the A-P extent of the arbor $(\boldsymbol{E})$, initial angle of growth $(\boldsymbol{F})$, percentage of axons that initially grow ventral but turn longitudinal $(\boldsymbol{G})$, percentage of axons that initially grow Iongitudinal but turn ventral $(\boldsymbol{H})$, number of branches $(\boldsymbol{I})$, and percentage of crossing over events $(\boldsymbol{J}) .{ }^{*} p<0.05,{ }^{* *} p<0.01$, ${ }^{* * *} p<0.005$. Numbers on graphs indicate number of axon arbors $(\boldsymbol{E}-\boldsymbol{I})$, or axon interactions $(\boldsymbol{J})$. Error bars indicate SEM.

$25.1 \pm 2.1$ branches $(p=0.03)$ (Fig. $6 I)$. These data suggest that the large embryo movements generated by muscle contractions are required to guide the peripheral $\mathrm{RB}$ axons.

chrna1 (nic-1) mutant embryos have reduced CSPG staining, but treatment with chondroitinase $\mathrm{ABC}$ does not cause peripheral $\mathrm{RB}$ axon guidance defects

Our initial characterization of the ttna (pik) mutants did not reveal changes in extracellular matrix molecules. However, this mutant has a milder axonal phenotype than mutants that completely block muscle contractions. To further test the possibility that muscle contractions act by altering the extracellular matrix, we analyzed matrix molecules in the most severely affected mutant, chrnal (nic-1). In the chrnal (nic-1) embryos, laminin immunostaining appeared normal (data not shown), suggesting that these mutants have an intact basal lamina between the muscles and the skin. In contrast, anti-CSPG labeling showed a marked reduction in the mutants. WT embryos show strong
CSPG labeling in the extracellular matrix surrounding the neural tube and the notochord (Fig. 7A, arrows), and weaker label under the skin where the peripheral RB axons grow (Fig. 7A, arrowhead). The CSPG staining in all areas was reduced in chrnal (nic-1) mutants (Fig. 7B, arrows and arrowhead).

CSPGs have previously been shown to function in guiding axons, often as inhibitory cues (Snow et al., 2001, 2003; Galtrey and Fawcett, 2007; Mizumoto et al., 2009), or by mediating axonal responses to other secreted guidance cues (Kantor et al., 2004; Chanana et al., 2009). They have also been implicated in guiding peripheral DRG (Cahoon and Scott, 1999) and RB (Somasekhar and Nordlander, 1997) axons in the skin. To test whether the CSPG reduction in chrnal (nic-1) embryos is responsible for the axon guidance errors, we treated WT embryos with chondroitinase $\mathrm{ABC}$ as an alternative means to remove CSPGs. This treatment reduced CSPG immunoreactivity to undetectable levels (Fig. 7C,D). However, these embryos did not show any obvious guidance defects in the peripheral RB axons (Fig. $7 F$ ) compared with controls (Fig. 7E). These results suggest the reduction in CSPG levels in chrnal (nic-1) mutants is not the primary cause of the axon guidance defects.

\section{Discussion}

In this study, we show that mechanical force caused by muscle contractions acts to guide sensory axons in vivo. We analyzed multiple mutants or treatments that affect muscle cells in different ways, including hedgehog signaling mutants that affect the differentiation of muscle fiber types and the guidance of motor axons, ttna (pik) mutants that have disrupted sarcomere organization, chrnal (nic-1) mutants and TC treatment that block the muscle response to $\mathrm{ACh}$, or agarose embedding that physically blocks embryo movements. The one common effect shared by all these manipulations is loss of muscle contractions or movement, suggesting that the RB axon phenotype is specifically caused by loss of muscle movements. Moreover, the degree to which embryo movement was inhibited correlated with the severity of axon defects. A possible explanation for this effect is that more extensive movements generate larger shear forces between muscle and skin, and thus greater effects on RB arbors with contacts on both these substrates. Interestingly, the RB neurons in the anterior-most spinal cord normally extend axons longitudinally over the hindbrain, in which there are no underlying muscle cells and thus no extensive movements. This represents a case in the WT embryo in which absence of underlying muscle contractions correlates with longitudinal peripheral RB axon extension, and supports the hypothesis that the mechanical force caused by muscle contractions guides the peripheral RB axons. 

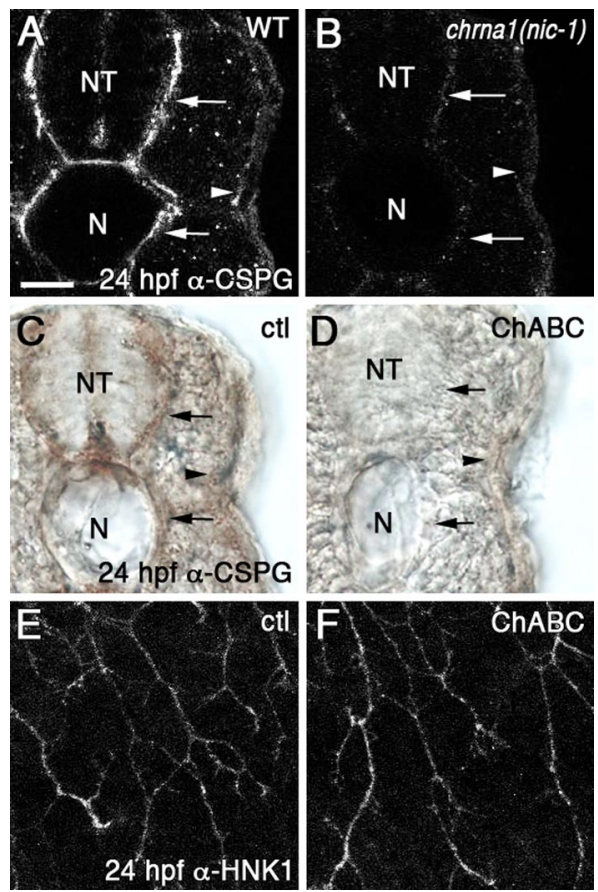

Figure 7. chrna1 (nic-1) mutants have reduced CSPG staining, but chondroitinase $A B C$ treatment does not cause peripheral RB axon defects. $A-D$, Cross sections of embryos at $24 \mathrm{hpf}$ showing CSPG immunostaining. $A$, WT embryos show CSPG label surrounding the neural tube (NT) and notochord (N) (arrows) and along the skin where the peripheral RB axons grow (arrowhead). $\boldsymbol{B}$, chrna1 (nic-1) embryos have reduced (SPG staining around the neural tube and notochord (arrows), and along the skin (arrowhead). C, Control (ctl)-treated embryos show normal CSPG staining around the neural tube and notochord (arrows) and skin (arrowhead). $D$, Embryos treated with chondroitinase $A B C$ (ChABC) have reduced or absent staining of CSPG (arrows and arrowhead).E, $\boldsymbol{F}$, Lateral views of embryos at $24 \mathrm{hpf}$ showing RB axons labeled with $\alpha$-HNK1. $\boldsymbol{E}$, Peripheral RB axons in control-treated embryos grow ventrally and avoid each other. $\boldsymbol{F}$, In ChABC-treated embryos, the axons appear normal. Scale bar, $20 \mu \mathrm{m}$.

We found that muscle contractions influence two aspects of $\mathrm{RB}$ axon guidance, ventral growth and mutual repulsion. It is not known to what degree these two behaviors might be mediated by common or separate molecular mechanisms. Our results are the first report of a manipulation affecting either of these processes, so it is unclear whether one mechanism affects the other. Although mutual repulsion between neighboring axons could serve to direct the axons ventrally by preventing extensive longitudinal growth, the timing and pattern of RB outgrowth suggests there is an additional mechanism promoting ventral growth. Because development proceeds in a general anterior-to-posterior gradient, $\mathrm{RB}$ axons at any given axial level initially extend without posterior neighbors. If contact repulsion were the only mechanism guiding axons ventrally, newly extending axons might be expected to turn toward the axon-free posterior direction. However, in WT embryos, these axons extend ventrally even without posterior neighbors to repel them. Thus, it is likely that additional signals guide the ventral trajectories of the axons, whereas contact repulsion shapes the final morphologies of the arbors (Sagasti et al., 2005). Since our results show that muscle contractions affect both ventral growth and contact repulsion, it is possible these processes converge on similar molecular pathways.

To date, nothing is known about the molecular mechanisms controlling either ventral growth or mutual repulsion of RB axons. However, recent studies have revealed some of the molecules that regulate other steps in $\mathrm{RB}$ axon guidance. The initial formation of the peripheral axon is dependent on LIM homeodomain transcription factors, such as Islet-2 (Segawa et al., 2001; Becker et al., 2002). Peripheral axons are guided out of the spinal cord in part by Semaphorin 3D (Liu and Halloran, 2005), and in Xenopus, peripheral axon extension within the skin is dependent on focal adhesion kinase activity (Robles and Gomez, 2006). Finally, secondary branching of peripheral arbors is controlled in part by Slit acting through Plexin-A4 (Miyashita et al., 2004; Yeo et al., 2004). Our results demonstrating a role for muscle contractions provide the first information about the mechanisms that control ventral growth and/or mutual repulsion.

\section{Ventral guidance of peripheral RB axons}

What might be the mechanism by which muscle contractions regulate ventral growth of $\mathrm{RB}$ axons? One possibility is that mechanical stimulation directly alters the direction of growth. Previous in vitro work has shown that axons change their direction of growth in response to mechanical manipulations (Bray, 1979; Anava et al., 2009). These studies suggest that axon branch growth and direction is governed by tension and the need to have an equilibrium of forces across the entire neuronal arbor. Thus, tension in one direction would cause axon growth in the opposite direction, thereby equalizing the forces. Muscle contraction may affect the peripheral RB axons in a similar manner. Muscle cells are aligned along the longitudinal axis, and the tension their contractions exert on RB axons thus would be predominantly longitudinal. $\mathrm{RB}$ axon growth in the ventral direction could serve to balance this longitudinal force.

Another possibility is that muscle contractions may act more indirectly by affecting a guidance cue that controls ventral growth of RB axons. For example, muscle contractions could stimulate stretch-activated channels on the muscles or the skin, which in turn could alter secretion or regulate localization of an extracellular guidance molecule expressed in a dorsal-ventral gradient. Interestingly, the mRNA expression of collagen $1 \alpha 2$ in the skin is altered in the chrna1 (nic-1) mutant (van der Meulen et al., 2005), although it is unknown whether collagen $1 \alpha 2$ affects peripheral $\mathrm{RB}$ axon direction. We also found that CSPG expression is reduced in chrnal (nic-1) mutants, suggesting that muscle activity can somehow affect CSPG levels. However, because chondroitinase $\mathrm{ABC}$ treatment did not alter axon pathways, the potential contribution of reduced CSPG levels to the axonal phenotype in chrnal (nic-1) is likely minimal. Although we cannot rule out the possibility that contraction is required for muscle cells to secrete an RB guidance cue, the agarose-embedding experiment argues against this possibility because agarose embedding only limits larger embryo movements, whereas individual muscle contractions can still occur. An alternative possibility is that the force of muscle contraction could alter the ability of axons to correctly sense and respond to a ventral guidance cue. Several second messengers that are important for determining the response of an axon to guidance cues, such as cAMP or calcium flow through TRP channels (Ming et al., 1997; Wang and Zheng, 1998; Li et al., 1999; Greka et al., 2003; Huber et al., 2003; Wang and Poo, 2005) also can be affected by mechanical stimulation (Chicurel et al., 1998; Meyer et al., 2000; Riveline et al., 2001; Nauli et al., 2003; Corey et al., 2004), consistent with this idea.

\section{Contact repulsion of peripheral $\mathrm{RB}$ axons}

Our live-imaging results show that defects in muscle contraction also lead to reduced mutual repulsion between peripheral RB axons. The molecules involved in mediating mutual repulsion are unknown; however, the Ig superfamily molecule Down syndrome cell adhesion molecule (DSCAM) has been shown to me- 
diate recognition and repulsion of neurites in other systems, such as dendritic tiling of Drosophila sensory neurons (Hughes et al., 2007; Matthews et al., 2007; Soba et al., 2007) and neurite arborization and mosaic spacing in mouse or chick retinal axons (Fuerst et al., 2008; Yamagata and Sanes, 2008). Thus, DSCAM might also be a candidate molecule to mediate contact repulsion in peripheral $\mathrm{RB}$ axons.

Our results suggest muscle movements influence the molecules mediating mutual repulsion or their signaling. We found that, although axons are not repelled in paralyzed embryos, they still appear to recognize each other, since they tend to grow along one another for short distances. This suggests that, without muscle contractions, the axons likely still express the cell surface molecules that mediate recognition and perhaps mutual repulsion. One possibility is that muscle contractions alter the levels or activities of downstream signaling molecules. These signaling molecules may or may not be the same ones that are necessary for ventral growth. It will be interesting to determine whether the molecular pathways that mediate contact repulsion converge on the same molecules that mediate ventral growth.

In conclusion, our results show that mechanical force generated by muscle contractions guides overlying sensory axons in vivo. Moreover, these results provide the first information on mechanisms controlling the ventral growth or contact repulsion in $\mathrm{RB}$ axons.

\section{References}

Ackerman KM, Nakkula R, Zirger JM, Beattie CE, Boyd RT (2009) Cloning and spatiotemporal expression of zebrafish neuronal nicotinic acetylcholine receptor alpha 6 and alpha 4 subunit RNAs. Dev Dyn 238:980-992.

Amsterdam A, Nissen RM, Sun Z, Swindell EC, Farrington S, Hopkins N (2004) Identification of 315 genes essential for early zebrafish development. Proc Natl Acad Sci U S A 101:12792-12797.

Anava S, Greenbaum A, Ben Jacob E, Hanein Y, Ayali A (2009) The regulative role of neurite mechanical tension in network development. Biophys J 96:1661-1670.

Balaban NQ, Schwarz US, Riveline D, Goichberg P, Tzur G, Sabanay I, Mahalu D, Safran S, Bershadsky A, Addadi L, Geiger B (2001) Force and focal adhesion assembly: a close relationship studied using elastic micropatterned substrates. Nat Cell Biol 3:466-472.

Barresi MJ, Stickney HL, Devoto SH (2000) The zebrafish slow-muscleomitted gene product is required for Hedgehog signal transduction and the development of slow muscle identity. Development 127:2189-2199.

Becker T, Ostendorff HP, Bossenz M, Schlüter A, Becker CG, Peirano RI, Bach I (2002) Multiple functions of LIM domain-binding CLIM/NLI/ Ldb cofactors during zebrafish development. Mech Dev 117:75-85.

Bernhardt RR, Chitnis AB, Lindamer L, Kuwada JY (1990) Identification of spinal neurons in the embryonic and larval zebrafish. J Comp Neurol 302:603-616.

Blader P, Plessy C, Strähle U (2003) Multiple regulatory elements with spatially and temporally distinct activities control neurogenin 1 expression in primary neurons of the zebrafish embryo. Mech Dev 120:211-218.

Brand M, Heisenberg CP, Warga RM, Pelegri F, Karlstrom RO, Beuchle D, Picker A, Jiang YJ, Furutani-Seiki M, van Eeden FJ, Granato M, Haffter P, Hammerschmidt M, Kane DA, Kelsh RN, Mullins MC, Odenthal J, Nüsslein-Volhard C (1996) Mutations affecting development of the midline and general body shape during zebrafish embryogenesis. Development 123:129-142.

Bray D (1979) Mechanical tension produced by nerve cells in tissue culture. J Cell Sci 37:391-410.

Bray D (1984) Axonal growth in response to experimentally applied mechanical tension. Dev Biol 102:379-389.

Cahoon SM, Scott SA (1999) Multiple mechanisms contribute to the avoidance of avian epidermis by sensory axons. Dev Biol 208:502-512.

Chan CE, Odde DJ (2008) Traction dynamics of filopodia on compliant substrates. Science 322:1687-1691.

Chanana B, Steigemann P, Jackle H, Vorbruggen G (2009) Reception of Slit requires only the chondroitin-sulphate-modified extracellular domain of
Syndecan at the target cell surface. Proc Natl Acad Sci U S A 106: 11984-11988.

Chicurel ME, Singer RH, Meyer CJ, Ingber DE (1998) Integrin binding and mechanical tension induce movement of mRNA and ribosomes to focal adhesions. Nature 392:730-733.

Chilton JK (2006) Molecular mechanisms of axon guidance. Dev Biol 292:13-24.

Clarke JD, Hayes BP, Hunt SP, Roberts A (1984) Sensory physiology, anatomy and immunohistochemistry of Rohon-Beard neurones in embryos of Xenopus laevis. J Physiol 348:511-525.

Corey DP, García-Añoveros J, Holt JR, Kwan KY, Lin SY, Vollrath MA, Amalfitano A, Cheung EL, Derfler BH, Duggan A, Géléoc GS, Gray PA, Hoffman MP, Rehm HL, Tamasauskas D, Zhang DS (2004) TRPA1 is a candidate for the mechanosensitive transduction channel of vertebrate hair cells. Nature 432:723-730.

Costa ML, Escaleira RC, Rodrigues VB, Manasfi M, Mermelstein CS (2002) Some distinctive features of zebrafish myogenesis based on unexpected distributions of the muscle cytoskeletal proteins actin, myosin, desmin, alpha-actinin, troponin and titin. Mech Dev 116:95-104.

Cox KH, DeLeon DV, Angerer LM, Angerer RC (1984) Detection of mRNAs in sea urchin embryos by in situ hybridization using asymmetric RNA probes. Dev Biol 101:485-502.

Etard C, Behra M, Ertzer R, Fischer N, Jesuthasan S, Blader P, Geisler R, Strähle U (2005) Mutation in the delta-subunit of the nAChR suppresses the muscle defects caused by lack of Dystrophin. Dev Dyn 234:1016-1025.

Feldner J, Becker T, Goishi K, Schweitzer J, Lee P, Schachner M, Klagsbrun M, Becker CG (2005) Neuropilin-1a is involved in trunk motor axon outgrowth in embryonic zebrafish. Dev Dyn 234:535-549.

Fuerst PG, Koizumi A, Masland RH, Burgess RW (2008) Neurite arborization and mosaic spacing in the mouse retina require DSCAM. Nature 451:470-474.

Galbraith CG, Yamada KM, Sheetz MP (2002) The relationship between force and focal complex development. J Cell Biol 159:695-705.

Gallo G, Letourneau PC (2004) Regulation of growth cone actin filaments by guidance cues. J Neurobiol 58:92-102.

Galtrey CM, Fawcett JW (2007) The role of chondroitin sulfate proteoglycans in regeneration and plasticity in the central nervous system. Brain Res Rev 54:1-18.

Gregg RG, Willer GB, Fadool JM, Dowling JE, Link BA (2003) Positional cloning of the young mutation identifies an essential role for the Brahma chromatin remodeling complex in mediating retinal cell differentiation. Proc Natl Acad Sci U S A 100:6535-6540.

Greka A, Navarro B, Oancea E, Duggan A, Clapham DE (2003) TRPC5 is a regulator of hippocampal neurite length and growth cone morphology. Nat Neurosci 6:837-845.

Grinblat Y, Lane ME, Sagerström C, Sive H (1999) Analysis of zebrafish development using explant culture assays. Methods Cell Biol 59:127-156.

Grunwald DJ, Kimmel CB, Westerfield M, Walker C, Streisinger G (1988) A neural degeneration mutation that spares primary neurons in the zebrafish. Dev Biol 126:115-128.

Haffter P, Granato M, Brand M, Mullins MC, Hammerschmidt M, Kane DA, Odenthal J, van Eeden FJ, Jiang YJ, Heisenberg CP, Kelsh RN, FurutaniSeiki M, Vogelsang E, Beuchle D, Schach U, Fabian C, Nüsslein-Volhard C (1996) The identification of genes with unique and essential functions in the development of the zebrafish, Danio rerio. Development 123:1-36.

Halloran MC, Berndt JD (2003) Current progress in neural crest cell motility and migration and future prospects for the zebrafish model system. Dev Dyn 228:497-513.

Halloran MC, Severance SM, Yee CS, Gemza DL, Raper JA, Kuwada JY (1999) Analysis of a zebrafish semaphorin reveals potential functions in vivo. Dev Dyn 214:13-25.

Hanneman E, Westerfield M (1989) Early expression of acetylcholinesterase activity in functionally distinct neurons of the zebrafish. J Comp Neurol 284:350-361.

Hatta K, Bremiller R, Westerfield M, Kimmel CB (1991) Diversity of expression of engrailed-like antigens in zebrafish. Development 112:821-832.

Heidemann SR, Buxbaum RE (1994) Mechanical tension as a regulator of axonal development. Neurotoxicology 15:95-107.

Honjo Y, Eisen JS (2005) Slow muscle regulates the pattern of trunk neural crest migration in zebrafish. Development 132:4461-4470.

Huber AB, Kolodkin AL, Ginty DD, Cloutier JF (2003) Signaling at the 
growth cone: ligand-receptor complexes and the control of axon growth and guidance. Annu Rev Neurosci 26:509-563.

Hughes ME, Bortnick R, Tsubouchi A, Bäumer P, Kondo M, Uemura T, Schmucker D (2007) Homophilic Dscam interactions control complex dendrite morphogenesis. Neuron 54:417-427.

Jiang FX, Yurke B, Firestein BL, Langrana NA (2008) Neurite outgrowth on a DNA crosslinked hydrogel with tunable stiffnesses. Ann Biomed Eng 36:1565-1579.

Kalil K, Dent EW (2005) Touch and go: guidance cues signal to the growth cone cytoskeleton. Curr Opin Neurobiol 15:521-526.

Kantor DB, Chivatakarn O, Peer KL, Oster SF, Inatani M, Hansen MJ, Flanagan JG, Yamaguchi Y, Sretavan DW, Giger RJ, Kolodkin AL (2004) Semaphorin $5 \mathrm{~A}$ is a bifunctional axon guidance cue regulated by heparan and chondroitin sulfate proteoglycans. Neuron 44:961-975.

Karlstrom RO, Trowe T, Klostermann S, Baier H, Brand M, Crawford AD, Grunewald B, Haffter P, Hoffmann H, Meyer SU, Müller BK, Richter S, van Eeden FJ, Nüsslein-Volhard C, Bonhoeffer F (1996) Zebrafish mutations affecting retinotectal axon pathfinding. Development 123: 427-438.

Karlstrom RO, Talbot WS, Schier AF (1999) Comparative synteny cloning of zebrafish you-too: mutations in the Hedgehog target gli2 affect ventral forebrain patterning. Genes Dev 13:388-393.

Kimmel CB, Ballard WW, Kimmel SR, Ullmann B, Schilling TF (1995) Stages of embryonic development of the zebrafish. Dev Dyn 203:253-310.

Kuwada JY, Bernhardt RR, Nguyen N (1990) Development of spinal neurons and tracts in the zebrafish embryo. J Comp Neurol 302:617-628.

Lamoureux P, Ruthel G, Buxbaum RE, Heidemann SR (2002) Mechanical tension can specify axonal fate in hippocampal neurons. J Cell Biol 159:499-508.

Lewis KE, Currie PD, Roy S, Schauerte H, Haffter P, Ingham PW (1999) Control of muscle cell-type specification in the zebrafish embryo by Hedgehog signalling. Dev Biol 216:469-480.

Li HS, Xu XZ, Montell C (1999) Activation of a TRPC3-dependent cation current through the neurotrophin BDNF. Neuron 24:261-273.

Liu DW, Westerfield M (1990) The formation of terminal fields in the absence of competitive interactions among primary motoneurons in the zebrafish. J Neurosci 10:3947-3959.

Liu Y, Halloran MC (2005) Central and peripheral axon branches from one neuron are guided differentially by Semaphorin $3 \mathrm{D}$ and transient axonal glycoprotein-1. J Neurosci 25:10556-10563.

Matthews BD, Overby DR, Mannix R, Ingber DE (2006) Cellular adaptation to mechanical stress: role of integrins, Rho, cytoskeletal tension and mechanosensitive ion channels. J Cell Sci 119:508-518.

Matthews BJ, Kim ME, Flanagan JJ, Hattori D, Clemens JC, Zipursky SL, Grueber WB (2007) Dendrite self-avoidance is controlled by Dscam. Cell 129:593-604.

Metcalfe WK, Myers PZ, Trevarrow B, Bass MB, Kimmel CB (1990) Primary neurons that express the L2/HNK-1 carbohydrate during early development in the zebrafish. Development 110:491-504.

Meyer CJ, Alenghat FJ, Rim P, Fong JH, Fabry B, Ingber DE (2000) Mechanical control of cyclic AMP signalling and gene transcription through integrins. Nat Cell Biol 2:666-668.

Ming G, Song H, Berninger B, Inagaki N, Tessier-Lavigne M, Poo M (1999) Phospholipase C-gamma and phosphoinositide 3-kinase mediate cytoplasmic signaling in nerve growth cone guidance. Neuron 23:139-148.

Ming GL, Song HJ, Berninger B, Holt CE, Tessier-Lavigne M, Poo MM (1997) cAMP-dependent growth cone guidance by netrin-1. Neuron 19:1225-1235.

Miyashita T, Yeo SY, Hirate Y, Segawa H, Wada H, Little MH, Yamada T, Takahashi N, Okamoto H (2004) PlexinA4 is necessary as a downstream target of Islet2 to mediate Slit signaling for promotion of sensory axon branching. Development 131:3705-3715.

Mizumoto S, Mikami T, Yasunaga D, Kobayashi N, Yamauchi H, Miyake A, Itoh N, Kitagawa H, Sugahara K (2009) Chondroitin 4-O-sulfotransferase-1 is required for somitic muscle development and motor axon guidance in zebrafish. Biochem J 419:387-399.

Muntz L (1975) Myogenesis in the trunk and leg during development of the tadpole of Xenopus laevis (Daudin 1802). J Embryol Exp Morphol 33:757-774.

Myers PZ, Eisen JS, Westerfield M (1986) Development and axonal outgrowth of identified motoneurons in the zebrafish. J Neurosci $6: 2278-2289$.
Nauli SM, Alenghat FJ, Luo Y, Williams E, Vassilev P, Li X, Elia AE, Lu W, Brown EM, Quinn SJ, Ingber DE, Zhou J (2003) Polycystins 1 and 2 mediate mechanosensation in the primary cilium of kidney cells. Nat Genet 33:129-137.

Paulus JD, Halloran MC (2006) Zebrafish bashful/laminin-alpha 1 mutants exhibit multiple axon guidance defects. Dev Dyn 235:213-224.

Pedersen SE, Cohen JB (1990) D-Tubocurarine binding sites are located at alpha-gamma and alpha-delta subunit interfaces of the nicotinic acetylcholine receptor. Proc Natl Acad Sci U S A 87:2785-2789.

Plopper G, Ingber DE (1993) Rapid induction and isolation of focal adhesion complexes. Biochem Biophys Res Commun 193:571-578.

Pollard SM, Parsons MJ, Kamei M, Kettleborough RN, Thomas KA, Pham VN, Bae MK, Scott A, Weinstein BM, Stemple DL (2006) Essential and overlapping roles for laminin alpha chains in notochord and blood vessel formation. Dev Biol 289:64-76.

Riveline D, Zamir E, Balaban NQ, Schwarz US, Ishizaki T, Narumiya S, Kam Z, Geiger B, Bershadsky AD (2001) Focal contacts as mechanosensors: externally applied local mechanical force induces growth of focal contacts by an mDia1-dependent and ROCK-independent mechanism. J Cell Biol 153:1175-1186.

Robles E, Gomez TM (2006) Focal adhesion kinase signaling at sites of integrin-mediated adhesion controls axon pathfinding. Nat Neurosci 9:1274-1283.

Sagasti A, Guido MR, Raible DW, Schier AF (2005) Repulsive interactions shape the morphologies and functional arrangement of zebrafish peripheral sensory arbors. Curr Biol 15:804-814.

Sato T, Mishina M (2003) Representational difference analysis, highresolution physical mapping, and transcript identification of the zebrafish genomic region for a motor behavior. Genomics 82:218-229.

Sato-Maeda M, Tawarayama H, Obinata M, Kuwada JY, Shoji W (2006) Sema3al guides spinal motor axons in a cell- and stage-specific manner in zebrafish. Development 133:937-947.

Schäfer M, Kinzel D, Winkler C (2007) Discontinuous organization and specification of the lateral floor plate in zebrafish. Dev Biol 301:117-129.

Schauerte HE, van Eeden FJ, Fricke C, Odenthal J, Strähle U, Haffter P (1998) Sonic hedgehog is not required for the induction of medial floor plate cells in the zebrafish. Development 125:2983-2993.

Schneider VA, Granato M (2006) The myotomal diwanka (lh3) glycosyltransferase and type XVIII collagen are critical for motor growth cone migration. Neuron 50:683-695.

Schweitzer J, Becker T, Lefebvre J, Granato M, Schachner M, Becker CG (2005) Tenascin-C is involved in motor axon outgrowth in the trunk of developing zebrafish. Dev Dyn 234:550-566.

Seeley M, Huang W, Chen Z, Wolff WO, Lin X, Xu X (2007) Depletion of zebrafish titin reduces cardiac contractility by disrupting the assembly of Z-discs and A-bands. Circ Res 100:238-245.

Segawa H, Miyashita T, Hirate Y, Higashijima S, Chino N, Uyemura K, Kikuchi Y, Okamoto H (2001) Functional repression of Islet-2 by disruption of complex with Ldb impairs peripheral axonal outgrowth in embryonic zebrafish. Neuron 30:423-436.

Sepich DS, Wegner J, O'Shea S, Westerfield M (1998) An altered intron inhibits synthesis of the acetylcholine receptor alpha-subunit in the paralyzed zebrafish mutant nic1. Genetics 148:361-372.

Snow DM, Mullins N, Hynds DL (2001) Nervous system-derived chondroitin sulfate proteoglycans regulate growth cone morphology and inhibit neurite outgrowth: a light, epifluorescence, and electron microscopy study. Microsc Res Tech 54:273-286.

Snow DM, Smith JD, Cunningham AT, McFarlin J, Goshorn EC (2003) Neurite elongation on chondroitin sulfate proteoglycans is characterized by axonal fasciculation. Exp Neurol 182:310-321.

Soba P, Zhu S, Emoto K, Younger S, Yang SJ, Yu HH, Lee T, Jan LY, Jan YN (2007) Drosophila sensory neurons require Dscam for dendritic selfavoidance and proper dendritic field organization. Neuron 54:403-416.

Somasekhar T, Nordlander RH (1997) Selective early innervation of a subset of epidermal cells in Xenopus may be mediated by chondroitin sulfate proteoglycans. Brain Res Dev Brain Res 99:208-215.

Steffen LS, Guyon JR, Vogel ED, Howell MH, Zhou Y, Weber GJ, Zon LI, Kunkel LM (2007) The zebrafish runzel muscular dystrophy is linked to the titin gene. Dev Biol 309:180-192.

Stemple DL, Solnica-Krezel L, Zwartkruis F, Neuhauss SC, Schier AF, Malicki J, Stainier DY, Abdelilah S, Rangini Z, Mountcastle-Shah E, Driever W 
(1996) Mutations affecting development of the notochord in zebrafish. Development 123:117-128.

Uemura O, Okada Y, Ando H, Guedj M, Higashijima S, Shimazaki T, Chino N, Okano H, Okamoto H (2005) Comparative functional genomics revealed conservation and diversification of three enhancers of the isl1 gene for motor and sensory neuron-specific expression. Dev Biol 278:587-606.

van der Meulen T, Schipper H, van Leeuwen JL, Kranenbarg S (2005) Effects of decreased muscle activity on developing axial musculature in nicb107 mutant zebrafish (Danio rerio). J Exp Biol 208:3675-3687.

van Eeden FJ, Granato M, Schach U, Brand M, Furutani-Seiki M, Haffter P, Hammerschmidt M, Heisenberg CP, Jiang YJ, Kane DA, Kelsh RN, Mullins MC, Odenthal J, Warga RM, Allende ML, Weinberg ES, NüssleinVolhard C (1996) Mutations affecting somite formation and patterning in the zebrafish, Danio rerio. Development 123:153-164.

Varga ZM, Amores A, Lewis KE, Yan YL, Postlethwait JH, Eisen JS, Westerfield M (2001) Zebrafish smoothened functions in ventral neural tube specification and axon tract formation. Development 128:3497-3509.

Wang GX, Poo MM (2005) Requirement of TRPC channels in netrin-1induced chemotropic turning of nerve growth cones. Nature 434:898904.

Wang Q, Zheng JQ (1998) cAMP-mediated regulation of neurotrophininduced collapse of nerve growth cones. J Neurosci 18:4973-4984.

Westerfield M, Liu DW, Kimmel CB, Walker C (1990) Pathfinding and synapse formation in a zebrafish mutant lacking functional acetylcholine receptors. Neuron 4:867-874.

Willer GB, Lee VM, Gregg RG, Link BA (2005) Analysis of the zebrafish perplexed mutation reveals tissue-specific roles for de novo pyrimidine synthesis during development. Genetics 170:1827-1837.

Willits RK, Skornia SL (2004) Effect of collagen gel stiffness on neurite extension. J Biomater Sci Polym Ed 15:1521-1531.

Wolman MA, Liu Y, Tawarayama H, Shoji W, Halloran MC (2004) Repulsion and attraction of axons by semaphorin3D are mediated by different neuropilins in vivo. J Neurosci 24:8428-8435.

Xu X, Meiler SE, Zhong TP, Mohideen M, Crossley DA, Burggren WW, Fishman MC (2002) Cardiomyopathy in zebrafish due to mutation in an alternatively spliced exon of titin. Nat Genet 30:205-209.

Yamagata M, Sanes JR (2008) Dscam and Sidekick proteins direct laminaspecific synaptic connections in vertebrate retina. Nature 451:465-469.

Yeo SY, Miyashita T, Fricke C, Little MH, Yamada T, Kuwada JY, Huh TL, Chien CB, Okamoto H (2004) Involvement of Islet-2 in the Slit signaling for axonal branching and defasciculation of the sensory neurons in embryonic zebrafish. Mech Dev 121:315-324.

Young HM, Anderson RB, Anderson CR (2004) Guidance cues involved in the development of the peripheral autonomic nervous system. Auton Neurosci 112:1-14.

Zeller J, Granato M (1999) The zebrafish diwanka gene controls an early step of motor growth cone migration. Development 126:3461-3472.

Zirger JM, Beattie CE, McKay DB, Boyd RT (2003) Cloning and expression of zebrafish neuronal nicotinic acetylcholine receptors. Gene Expr Patterns 3:747-754.

Zou Y, Lyuksyutova AI (2007) Morphogens as conserved axon guidance cues. Curr Opin Neurobiol 17:22-28. 